\title{
The Study Spectral Analysis To Random Vibrations For Nonlinear Oscillators
}

\author{
P Stan", M Stan \\ University Of Pitesti, Arges, Romania \\ *Corresponding author e-mail: petre.stan@upit.ro \\ Article history \\ Received 20.07.2017 \\ Accepted 21.09.2017 \\ DOI https://doi.org/10.26825/bup.ar.2017.005
}

\begin{abstract}
In the theory of random vibrations, some basic principles of the probability theory are applied. Random vibration can be represented in the frequency domain in terms of a Fourier transform or Power Spectral Density Function. In this paper we will study the nonlinear vibration of an oscillator by determining the spectral density of response. We will present the corresponding numerical results and the spectral density diagrams of the response according to the frequency. The method will be briefly discussed in the following section.
\end{abstract}

\section{Introduction}

The demand for engineering structures is continuously increasing. The study of random vibration problems is a relatively new engineering discipline. Examples include the response of buildings to wind loading and earthquakes, the vibration of vehicles travelling over rough ground and the excitation on aircraft by atmospheric turbulence and jet noise. It was difficult to find the exact or closed-form solutions for nonlinear problems. Nonlinear systems can display behaviours that linear systems cannot. A natural method of non-linear problems is to replace governing set of non-linear equations by an equivalent set of linear equations. The difference between the sets being minimized in some appropriate sense.We present a method for estimating the power spectral density of the stationary response of oscillator with a nonlinear restoring force under external stochastic wideband excitation. An equivalent linear system is derived, from which the power spectral density is deduced. 


\section{The system model}

For the presentation of the statistical linearization method, we will consider a system consisting of $\mathrm{n}$ oscillators with a nonlinear restoring force component. The equations of motion of the n-degreeof-freedom system are given by:

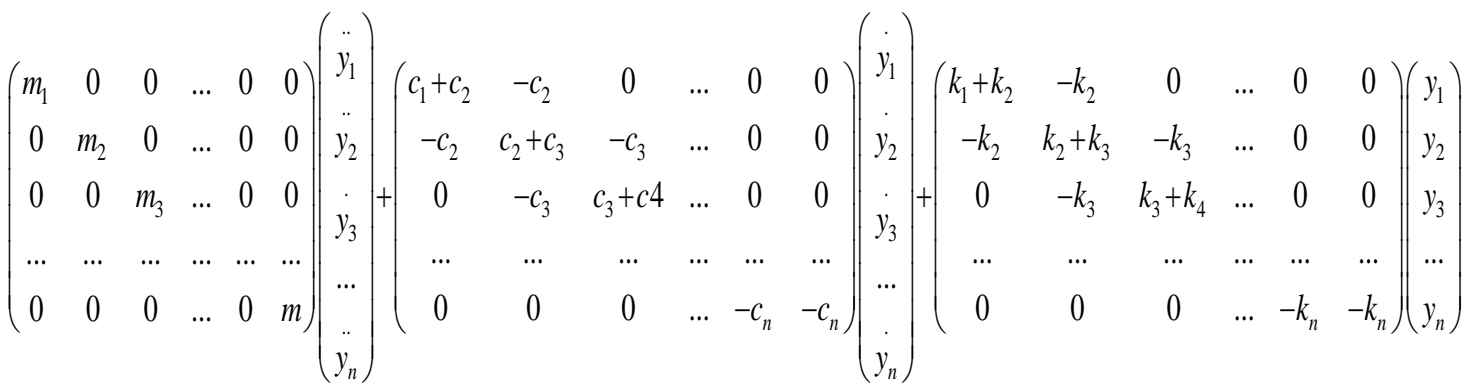

$$
\begin{aligned}
& +\left(\begin{array}{cccccc}
k_{1}+k_{2} & -k_{2} & 0 & \ldots & 0 & 0 \\
-k_{2} & k_{2}+k_{3} & -k_{3} & \ldots & 0 & 0 \\
0 & -k_{3} & k_{3}+k_{4} & \ldots & 0 & 0 \\
\ldots & \ldots & \ldots & \ldots & \ldots & \ldots \\
0 & 0 & 0 & \ldots & -k_{n} & -k_{n}
\end{array}\right)\left(\sum_{i=2}^{n} a_{i} y_{1}{ }^{i} \sum_{i=2}^{n} a_{i} y_{2}{ }^{i} \sum_{i=2}^{n} a_{i} y_{3}{ }^{i} \quad \ldots \sum_{i=2}^{n} a_{i} y_{n}{ }^{i}\right)^{\mathrm{t}}=\left(\begin{array}{c}
F(t) \\
0 \\
0 \\
\ldots \\
0
\end{array}\right)
\end{aligned}
$$

where $\mathrm{m}_{1}, \mathrm{~m}_{2}, \ldots, \mathrm{m}_{\mathrm{n}}$ are the masses of the system, $\mathrm{c}_{1}, \mathrm{c}_{2}, \ldots, \mathrm{c}_{\mathrm{n}}$ are the viscous damping coefficients, $F(t)$ is the external excitation signal with zero mean, $y(t)=\left(\begin{array}{lllll}y_{1} & y_{2} & y_{3} & \ldots & y_{n}\end{array}\right)^{t}$ is the displacement response of the system and the component nonlinear is of the form: $[A]=\left(\sum_{i=2}^{n} a_{i} y_{1}{ }^{i} \quad \sum_{i=2}^{n} a_{i} y_{2}{ }^{i} \quad \sum_{i=2}^{n} a_{i} y_{3}{ }^{i} \quad \ldots \quad \sum_{i=2}^{n} a_{i} y_{n}{ }^{i}\right)^{T}$. The nonlinear factor $\mathrm{a}_{1}, \mathrm{a}_{2}, \ldots, \mathrm{a}_{\mathrm{n}}$ controls the type and degree of nonlinearity in the system. A higher value of $a_{1}, a_{2}, \ldots, a_{n}$ indicates a stronger nonlinearity. A positive value of $a_{1}, a_{2}, \ldots, a_{n}$ represents a hardening system while a negative value represents a softening system behavior. The linear equation [1] can be write:

$$
\begin{aligned}
& \left(\begin{array}{cccccc}
m_{1} & 0 & 0 & \ldots & 0 & 0 \\
0 & m_{2} & 0 & \ldots & 0 & 0 \\
0 & 0 & m_{3} & \ldots & 0 & 0 \\
\ldots & \ldots & \ldots & \ldots & \ldots & \ldots \\
0 & 0 & 0 & \ldots & 0 & m
\end{array}\right)\left(\begin{array}{c}
. . \\
y_{1} \\
\ddot{y_{2}} \\
y_{3} \\
\ldots \\
y_{n}
\end{array}\right)+\left(\begin{array}{cccccc}
c_{1}+c_{2} & -c_{2} & 0 & \ldots & 0 & 0 \\
-c_{2} & c_{2}+c_{3} & -c_{3} & \ldots & 0 & 0 \\
0 & -c_{3} & c_{3}+c 4 & \ldots & 0 & 0 \\
\ldots & \ldots & \ldots & \ldots & \ldots & \ldots \\
0 & 0 & 0 & \ldots & -c_{n} & -c_{n}
\end{array}\right)\left(\begin{array}{c}
y_{1} \\
y_{2} \\
y_{3} \\
\ldots \\
y_{n}
\end{array}\right)+\left(\begin{array}{cccccc}
c_{1}+c_{2} & -c_{2} & 0 & \ldots & 0 & 0 \\
-c_{2} & c_{2}+c_{3} & -c_{3} & \ldots & 0 & 0 \\
0 & -c_{3} & c_{3}+c 4 & \ldots & 0 & 0 \\
\ldots & \ldots & \ldots & \ldots & \ldots & \ldots \\
0 & 0 & 0 & \ldots & -c_{n} & -c_{n}
\end{array}\right)\left(\begin{array}{c}
\dot{y_{1}} \\
y_{1} \\
y_{2} \\
y_{3} \\
y_{n}
\end{array}\right) \\
& +\left(\begin{array}{cccccc}
m_{1} & 0 & 0 & \ldots & 0 & 0 \\
0 & m_{2} & 0 & \ldots & 0 & 0 \\
0 & 0 & m_{3} & \ldots & 0 & 0 \\
\ldots & \ldots & \ldots & \ldots & \ldots & \ldots \\
0 & 0 & 0 & \ldots & 0 & m
\end{array}\right)\left(\begin{array}{c}
\ddot{y_{1}} \\
\ddot{y_{2}} \\
y_{3} \\
y_{3} \\
y_{n}
\end{array}\right)+\left(\begin{array}{cccccc}
k_{1 e}+k_{2 e} & -k_{2 e} & 0 & \ldots & 0 & 0 \\
-k_{2 e} & k_{2 e}+k_{3 e} & -k_{3 e} & \ldots & 0 & 0 \\
0 & -k_{3 e} & k_{3 e}+k_{4 e} & \ldots & 0 & 0 \\
\ldots & \ldots & \ldots & \ldots & \ldots & \ldots \\
0 & 0 & 0 & \ldots & -k_{n e} & -k_{n e}
\end{array}\right)\left(\begin{array}{c}
y_{1} \\
y_{2} \\
y_{3} \\
\ldots \\
y_{n}
\end{array}\right)=\left(\begin{array}{c}
F(t) \\
0 \\
0 \\
0 \\
\ldots \\
0
\end{array}\right) .
\end{aligned}
$$


The difference between the nonlinear stiffness and linear stiffness terms [1] is $e=\left(\begin{array}{lllll}e_{1} & e_{2} & e_{3} & \ldots & e_{n}\end{array}\right)^{t}$,

$$
\begin{aligned}
& e_{1}=\left(k_{1}+k_{2}\right) y_{1}-k_{2} y_{2}+\left(k_{1}+k_{2}\right) \sum_{i=2}^{n} a_{i} y_{1}{ }^{i}-k_{2} \sum_{i=2}^{n} a_{i} y_{2}{ }^{i}+\left(k_{1 e}+k_{2 e}\right) y_{1}+k_{2 e} y_{2} \\
& e_{2}=-k_{2} y_{1}+\left(k_{2}+k_{3}\right) y_{2}-k_{3} y_{3}-k_{2} \sum_{i=2}^{n} a_{i} y_{1}{ }^{i}+\left(k_{2}+k_{3}\right) \sum_{i=2}^{n} a_{i} y_{2}{ }^{i}-k_{3} \sum_{i=2}^{n} a_{i} y_{3}{ }^{i}-k_{2 e} y_{1}-\left(k_{2 e}+k_{3 e}\right) y_{2} \\
& e_{3}=-k_{3} y_{2}+\left(k_{3}+k_{4}\right) y_{3}-k_{4} y_{4}-k_{3} \sum_{i=2}^{n} a_{i} y_{2}{ }^{i}+\left(k_{3}+k_{4}\right) \sum_{i=2}^{n} a_{i} y_{3}{ }^{i}-k_{4} \sum_{i=2}^{n} a_{i} y_{4}{ }^{i}-k_{3 e} y_{2}-\left(k_{3 e}+k_{4 e}\right) y_{3}+k_{4 e} y_{4}
\end{aligned}
$$

$$
e_{n-1}=-k_{n-1} y_{n-2}+\left(k_{n-1}+k_{n}\right) y_{n-1}-k_{n} y_{n}-k_{n-1} \sum_{i=2}^{n} a_{i} y_{n-2}{ }^{i}+\left(k_{n-1}+k_{n}\right) \sum_{i=2}^{n} a_{i} y_{n-1}{ }^{i}-k_{n} \sum_{i=2}^{n} a_{i} y_{n}{ }^{i}-k_{(n-1) e} y_{n-2}-\left(k_{(n-1) e}+k_{n e}\right) y_{n-1}+k_{n e} y_{n}(
$$

$$
e_{n}=-k_{n} y_{n-1}+k_{n} y_{n}-k_{n} \sum_{i=2}^{n} a_{i} y_{n-1}{ }^{i}+k_{n} \sum_{i=2}^{n} a_{i} y_{n}{ }^{i}+k_{n e} y_{n-1}+k_{n e} y_{n} \text {. }
$$

$\mathrm{yn}(\mathrm{t})$

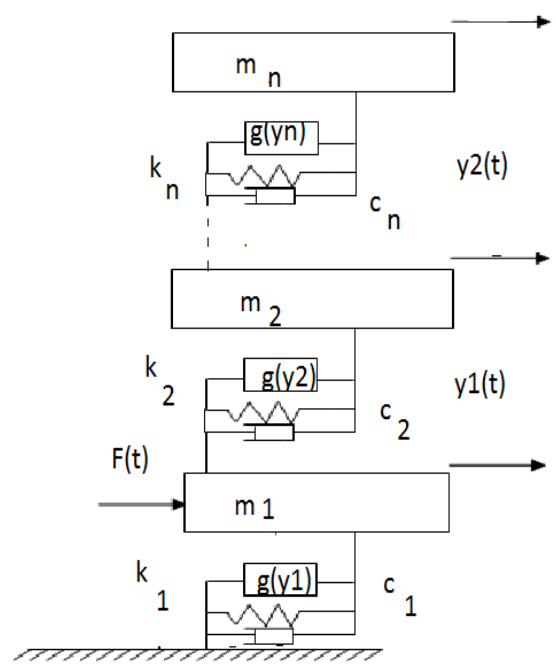

Figure 1. Analytical model.

$$
\begin{aligned}
& E\left\{e_{1}^{2}\right\}=\left(k_{1}+k_{2}\right)^{2} E\left\{y_{1}{ }^{2}\right\}+k_{2}{ }^{2} E\left\{y_{2}{ }^{2}\right\}+\left(k_{1}+k_{2}\right)^{2} E\left\{\left(\sum_{i=2}^{n} a_{i} y_{1}{ }^{i}\right)^{2}\right\}+k_{2}{ }^{2} E\left\{\left(\sum_{i=2}^{n} a_{i} y_{2}{ }^{i}\right)^{2}\right\}+\left(k_{1 e}+k_{2 e}\right)^{2} E\left\{y_{1}{ }^{2}\right\}+k_{2 e}{ }^{2} E\left\{y_{2}{ }^{2}\right\} \\
& -2\left(k_{1}+k_{2}\right) k_{2} E\left\{\eta_{2} \sum_{i=2}^{n} a_{i} y_{1}{ }^{i}+2 k_{2}{ }^{2} E\left\{y_{2} \sum_{i=2}^{n} a_{i} y_{2}{ }^{i}\right\}+2\left(k_{1 e}+k_{2 e}\right) k_{2} E\left\{y_{1} y_{2}\right\}-2 k_{2} k_{2 e} E\left\{y_{2}{ }^{2}\right\}\right.
\end{aligned}
$$




$$
\begin{aligned}
& -2\left(k_{1}+k_{2}\right) k_{2} E\left\{\sum_{i=2}^{n} a_{i} y_{1}{ }^{i} \sum_{i=2}^{n} a_{i} y_{2}{ }^{i}\right\}-2\left(k_{1}+k_{2}\right) k_{2} E\left\{y_{1} y_{2}\right\}+2\left(k_{1}+k_{2}\right)^{2} E\left\{y_{1} \sum_{i=2}^{n} a_{i} y_{1}{ }^{i}\right\} \\
& -2\left(k_{1}+k_{2}\right) k_{2} E\left\{y_{1} \sum_{i=2}^{n} a_{i} y_{2}{ }^{i}\right\}-2\left(k_{1}+k_{2}\right)\left(k_{1 e}+k_{2 e}\right) E\left\{y_{1}{ }^{2}\right\}+2\left(k_{1}+k_{2}\right) k_{2 e} E\left\{\eta_{1} \eta_{2}\right\}-2\left(k_{1}+k_{2}\right)\left(k_{1 e}\right. \\
& \left.+k_{2 e}\right) E\left\{y_{1} \sum_{i=2}^{n} a_{i} y_{1}{ }^{i}\right\}+\left(k_{1}+k_{2}\right) k_{2 e} E\left\{y_{2} \sum_{i=2}^{n} a_{i} y_{1}{ }^{i}\right\}+2\left(k_{1 e}+k_{2 e}\right) k_{2} E\left\{y_{1} \sum_{i=2}^{n} a_{i} y_{2}{ }^{i}\right\} \\
& -2 k_{2} k_{2 e} E\left\{y_{2} \sum_{i=2}^{n} a_{i} y_{2}{ }^{i}\right\}-2\left(k_{1 e}+k_{2 e}\right) k_{2 e} E\left\{y_{1} y_{2}\right\} .
\end{aligned}
$$

$$
\begin{aligned}
& E\left\{e_{n-1}^{2}\right\}=k_{n-1}^{2} E\left\{y_{n-1}{ }^{2}\right\}+\left(k_{n-1}+k_{n}\right)^{2} E\left\{y_{n-1}{ }^{2}\right\}+k_{n}^{2} E\left\{y_{n}{ }^{2}\right\}+k_{n-1}^{2} E\left\{\left(\sum_{i=2}^{n} a_{i} y_{n-2}{ }^{i}\right)^{2}\right\}+\left(k_{n-1}+k_{n}\right)^{2} E\left\{\left(\sum_{i=2}^{n} a_{i} y_{n-1}{ }^{i}\right)^{2}\right\} \\
& +k_{n}^{2} E\left\{\left(\sum_{i=2}^{n} a_{i} y_{n}^{i}\right)^{2}\right\}+k_{n-1}^{2} E\left\{y_{n-2}{ }^{2}\right\}+\left(k_{(n-1) e}+k_{n e}\right)^{2} E\left\{y_{n-1}{ }^{2}\right\}+k_{n e}{ }^{2} E\left\{y_{n}{ }^{2}\right\} \\
& -2\left(k_{n-1}+k_{n}\right) k_{n-1} E\left\{y_{n-2} y_{n-1}\right\}+2 k_{n-1} k_{n} E\left\{y_{n-2} y_{n}\right\}+2 k_{n-1}{ }^{2} E\left\{y_{1} \sum_{i=2}^{n} a_{i} y_{1}{ }^{i}\right\} \\
& -2 k_{n-1}\left(k_{n-1}+k_{n}\right) E\left\{y_{n-2} \sum_{i=2}^{n} a_{i} y_{n-2}{ }^{i}\right\}+2 k_{n-1} k_{n} E\left\{y_{n-2} \sum_{i=2}^{n} a_{i} y_{n}{ }^{i}\right\}+2 k_{n-1} k_{(n-1) e} E\left\{y_{n-2}{ }^{2}\right\} \\
& +2 k_{n-1}\left(k_{(n-1) e}+k_{n e}\right) E\left\{y_{n-2} y_{n-1}\right\}-2 k_{n-1} k_{n e} E\left\{y_{n-2} y_{n}\right\}-2 k_{n}\left(k_{n-1}+k_{n}\right) E\left\{y_{2} y_{3}\right\}-2 k_{n-1}\left(k_{n-1}\right. \\
& \left.+k_{n}\right) E\left\{y_{n-1} \sum_{i=2}^{n} a_{i} y_{n-2}{ }^{i}\right\}+2\left(k_{n-1}+k_{n}\right)^{2} E\left\{y_{n-1} \sum_{i=2}^{n} a_{i} y_{n-1}{ }^{i}\right\}-2 k_{n}\left(k_{n-1}+k_{n}\right) E\left\{y_{n-1} \sum_{i=2}^{n} a_{i} y_{n}{ }^{i}\right\}-2 k_{(n-1) e}\left(k_{n-1}\right. \\
& \left.+k_{n}\right) E\left\{y_{n-2} y_{n-1}\right\}-2\left(k_{n-1}+k_{n}\right)\left(k_{(n-1) e}+k_{n e}\right) E\left\{y_{n-1}^{2}\right\}+2\left(k_{2}+k_{3}\right) k_{n e} E\left\{y_{n-1} y_{n}\right\} \\
& +2 k_{n-1} k_{n} E\left\{y_{n} \sum_{i=2}^{n} a_{i} y_{n-2}{ }^{i}\right\}-2 k_{n}\left(k_{n-1}+k_{n}\right) E\left\{y_{n} \sum_{i=2}^{n} a_{i} y_{n-1}{ }^{i}\right\}+2 k_{n}{ }^{2} E\left\{y_{n} \sum_{i=2}^{n} a_{i} y_{n}{ }^{i}\right\} \\
& +2 k_{n} k_{(n-1) e} E\left\{y_{n-2} y_{n}\right\}+2 k_{n}\left(k_{(n-1) e}+k_{n e}\right) E\left\{y_{n-1} y_{n}\right\}-2 k_{n} k_{n e} E\left\{y_{n}^{2}\right\}-2 k_{n-1}\left(k_{n-1}\right. \\
& \left.+k_{n}\right) E\left\{\sum_{i=2}^{n} a_{i} y_{n-2}{ }^{i} \sum_{i=2}^{n} a_{i} y_{n-1}{ }^{i}\right\}+2 k_{n-1} k_{n} E\left\{\sum_{i=2}^{n} a_{i} y_{n-2}{ }^{i} \sum_{i=2}^{n} a_{i} y_{n}{ }^{i}\right\}+2 k_{n-1} k_{(n-1) e} E\left\{y_{n-2} \sum_{i=2}^{n} a_{i} y_{n-2}{ }^{i}\right\} \\
& +2 k_{n-1} k_{(n-1) e} E\left\{y_{n-1} \sum_{i=2}^{n} a_{i} y_{n-2}{ }^{i}\right\}-2 k_{n-1} k_{n e} E\left\{y_{n} \sum_{i=2}^{n} a_{i} y_{n-2}{ }^{i}\right\}-2 k_{n}\left(k_{n-1}\right. \\
& \left.+k_{n}\right) E\left\{\sum_{i=2}^{n} a_{i} y_{n-1}{ }^{i} \sum_{i=2}^{n} a_{i} y_{n}{ }^{i}\right\}-2 k_{(n-1) e}\left(k_{n-1}+k_{n}\right) E\left\{\sum_{i=2}^{n} a_{i} y_{n-1}{ }^{i}\right\}-2 k_{n-1}\left(k_{n-1}\right. \\
& \left.+k_{n}\right)\left(k_{(n-1) e}+k_{n e}\right) E\left\{y_{n-1} \sum_{i=2}^{n} a_{i} y_{n-1}{ }^{i}\right\}+2 k_{n e}\left(k_{n-1}+k_{n}\right) E\left\{y_{n} \sum_{i=2}^{n} a_{i} y_{n-1}{ }^{i}\right\} \\
& +2 k_{n} k_{(n-1) e} E\left\{y_{n-2} \sum_{i=2}^{n} a_{i} y_{n}{ }^{i}\right\}+2 k_{n}\left(k_{(n-1) e}+k_{n e}\right) E\left\{y_{n-1} \sum_{i=2}^{n} a_{i} y_{n}{ }^{i}\right\}-2 k_{n} k_{n e} E\left\{y_{n} \sum_{i=2}^{n} a_{i} y_{n}{ }^{i}\right\} \\
& +2 k_{(n-1) e}\left(k_{(n-1) e}+k_{n}\right) E\left\{y_{n-2} y_{n-1}\right\}-2 k_{(n-1) e} k_{n e} E\left\{y_{n-2} y_{n}\right\}-2\left(k_{(n-1) e}+k_{n e}\right) E\left\{y_{n-1} y_{n}\right\}
\end{aligned}
$$




$$
\begin{aligned}
& E\left\{e_{n}{ }^{2}\right\}=k_{n}{ }^{2} E\left\{y_{n-1}{ }^{2}\right\}+k_{n}{ }^{2} E\left\{y_{n}{ }^{2}\right\}+k_{n}{ }^{2} E\left\{\left(\sum_{i=2}^{n} a_{i} y_{n-1}{ }^{i}\right)^{2}\right\}+k_{n}{ }^{2} E\left\{\left(\sum_{i=2}^{n} a_{i} y_{n}{ }^{i}\right)^{2}\right\}+k_{n e}{ }^{2} E\left\{y_{n-1}{ }^{2}\right\}+k_{n e}{ }^{2} E\left\{y_{n}{ }^{2}\right\} \\
& -2 k_{n}{ }^{2} E\left\{y_{n-1} y_{n}\right\}+2 k_{n}{ }^{2} E\left\{y_{n-1} \sum_{i=2}^{n} a_{i} y_{n-1}{ }^{i}\right\}-2 k_{n}{ }^{2} E\left\{y_{n-1} \sum_{i=2}^{n} a_{i} y_{n}{ }^{i}\right\}-2 k_{n} k_{n e} E\left\{y_{n-1}{ }^{2}\right\} \\
& -2 k_{n} k_{n e} E\left\{y_{n-1} y_{n}\right\}-2 k_{n}{ }^{2} E\left\{y_{n} \sum_{i=2}^{n} a_{i} y_{n-1}{ }^{i}\right\}+2 k_{n}{ }^{2} E\left\{y_{n} \sum_{i=2}^{n} a_{i} y_{n}{ }^{i}\right\}+2 k_{n} k_{n e} E\left\{y_{n-1} y_{n}\right\} \\
& +2 k_{n} k_{n e} E\left\{y_{n}{ }^{2}\right\}-2 k_{n} k_{n e} E\left\{y_{n-1} \sum_{i=2}^{n} a_{i} y_{n-1}{ }^{i}\right\}-2 k_{n}{ }^{2} E\left\{\sum_{i=2}^{n} a_{i} y_{n-1}{ }^{i} \sum_{i=2}^{n} a_{i} y_{n}{ }^{i}\right\}-2 k_{n} k_{n e} \\
& \cdot E\left\{y_{n} \sum_{i=2}^{n} a_{i} y_{n-1}{ }^{i}\right\}+2 k_{n} k_{n e} E\left\{y_{n-1} \sum_{i=2}^{n} a_{i} y_{n}{ }^{i}\right\}+2 k_{n} k_{n e} E\left\{y_{n} \sum_{i=2}^{n} a_{i} y_{n}{ }^{i}\right\}+2 k_{n e}{ }^{2} E\left\{y_{n-1} y_{n}\right\}
\end{aligned}
$$

The value of $k_{1 e}, k_{2 e}, \ldots, k_{n e}$ can be obtained [1] by minimizing the expectation [2] of the square error:

$$
\frac{d E\left\{e_{1}^{2}\right\}}{d k_{1 e}}=0, \quad \frac{d E\left\{e_{2}^{2}\right\}}{d k_{2 e}}=0, \ldots, \frac{d E\left\{e_{n}^{2}\right\}}{d k_{n e}}=0 .
$$

We will get the following system of equations [3], resulting in the values of the parameters $k_{1 e}, \ldots, k_{n e}$ :

$$
2\left(k_{1 e}+k_{2 e}\right) E\left\{y_{1}{ }^{2}\right\}-2\left(k_{1}+k_{2}\right) E\left\{y_{1}{ }^{2}\right\}+2 k_{2} E\left\{\eta_{1} \eta_{2}\right\}-2\left(k_{1}+k_{2}\right) E\left\{y_{1} \sum_{i=2}^{n} a_{i} y_{1}{ }^{i}\right\}+2 k_{2} E\left\{y_{1} \sum_{i=2}^{n} a_{i} y_{2}{ }^{i}\right\}-2 k_{2 e} E\left\{y_{1} y_{2}\right\}=0(
$$

$$
\begin{aligned}
& 2\left(k_{1 e}+k_{2 e}\right) E\left\{y_{2}{ }^{2}\right\}+2 k_{2} E\left\{y_{1} y_{2}\right\}-2\left(k_{2}+k_{3}\right) E\left\{y_{1} y_{2}\right\}-2\left(k_{2}+k_{3}\right) E\left\{y_{2}{ }^{2}\right\}+2 k_{3} E\left\{y_{1} y_{3}\right\} \\
& +2 k_{3} E\left\{y_{2} y_{3}\right\}+2 k_{2} E\left\{y_{1} \sum_{i=2}^{n} a_{i} y_{1}{ }^{i}\right\}+2 k_{2} E\left\{y_{2} \sum_{i=2}^{n} a_{i} y_{1}{ }^{i}\right\}-2\left(k_{2}+k_{3}\right) E\left\{\sum_{i=2}^{n} a_{i} y_{2}{ }^{i}\right\}-2 k_{2}\left(k_{2}\right. \\
& \left.+k_{3}\right) E\left\{y_{2} \sum_{i=2}^{n} a_{i} y_{2}{ }^{i}\right\}+2 k_{3} E\left\{y_{1} \sum_{i=2}^{n} a_{i} y_{3}{ }^{i}\right\}+2 k_{3} E\left\{y_{2} \sum_{i=2}^{n} a_{i} y_{3}{ }^{i}\right\}+2\left(2 k_{2 e}\right. \\
& \left.+k_{3 e}\right) E\left\{y_{1} y_{2}\right\}-2 k_{3 e} E\left\{y_{1} y_{3}\right\}-2 E\left\{y_{2} y_{3}\right\}=0 \\
& 2\left(k_{(n-1) e}+k_{n e}\right) E\left\{y_{n-1}{ }^{2}\right\}+2 k_{n e} E\left\{y_{n}{ }^{2}\right\}+2 k_{n-1} E\left\{y_{n-2}{ }^{2}\right\}+2 k_{n-1} E\left\{y_{n-2} y_{n-1}\right\}-2\left(k_{n-1}+k_{n}\right) E\left\{y_{n-2} y_{n-1}\right\}-2\left(k_{n-1}\right. \\
& \left.+k_{n}\right) E\left\{y_{n-1}{ }^{2}\right\}+2 k_{n} k_{(n-1) e} E\left\{y_{n-2} y_{n}\right\}+2 k_{n}\left(k_{(n-1) e}+k_{n e}\right) E\left\{y_{n-1} y_{n}\right\}+2 k_{n-1} E\left\{y_{n-2}\right. \\
& \left.\cdot \sum_{i=2}^{n} a_{i} y_{n-2}{ }^{i}\right\}+2 k_{n-1} E\left\{y_{n-1} \sum_{i=2}^{n} a_{i} y_{n-2}{ }^{i}\right\}-2\left(k_{n-1}+k_{n}\right) E\left\{\sum_{i=2}^{n} a_{i} y_{n-1}{ }^{i}\right\}-2 k_{n-1}\left(k_{n-1}\right. \\
& \left.+k_{n}\right) E\left\{y_{n-1} \sum_{i=2}^{n} a_{i} y_{n-1}{ }^{i}\right\}+2 k_{n} E\left\{y_{n-2} \sum_{i=2}^{n} a_{i} y_{n}{ }^{i}\right\}+2 k_{n} E\left\{y_{n-1} \sum_{i=2}^{n} a_{i} y_{n}{ }^{i}\right\}+2\left(2 k_{(n-1) e}\right. \\
& \left.+2 k_{n}\right) E\left\{y_{n-2} y_{n-1}\right\}-2 k_{n e} E\left\{y_{n-2} y_{n}\right\}-2 E\left\{y_{n-1} y_{n}\right\}=0 \\
& 2 k_{n e} E\left\{y_{n-1}{ }^{2}\right\}+2 k_{n e} E\left\{y_{n}{ }^{2}\right\}-2 k_{n} E\left\{y_{n-1}{ }^{2}\right\}-2 k_{n} E\left\{y_{n-1} y_{n}\right\}+2 k_{n} E\left\{y_{n-1} y_{n}\right\}+2 k_{n} E\left\{y_{n}{ }^{2}\right\}-2 k_{n} E\left\{y_{n-1} \sum_{i=2}^{n} a_{i} y_{n-1}{ }^{i}\right\} \\
& -2 k_{n} E\left\{y_{n} \sum_{i=2}^{n} a_{i} y_{n-1}{ }^{i}\right\}+2 k_{n} E\left\{y_{n-1} \sum_{i=2}^{n} a_{i} y_{n}{ }^{i}\right\}+2 k_{n} E\left\{y_{n} \sum_{i=2}^{n} a_{i} y_{n}{ }^{i}\right\}+4 k_{n e} E\left\{y_{n-1} y_{n}\right\}=0 .
\end{aligned}
$$

Using the Fourier transform [4] of equation (2) and having the relations:

$$
f\left(\dot{y}_{j}(t)\right)=i \omega \bar{y}_{j}(\omega), \quad F\left(\ddot{y}_{j}(t)\right)=i \omega f\left(\dot{y}_{j}(t)\right)=-\omega^{2} \bar{y}_{j}(\omega), F((F(t))=\bar{F}(\omega),
$$

obtain the sistem: 


$$
\left\{\begin{array}{l}
\bar{y}_{1}(\omega)\left[-m_{1} \omega^{2}+k_{1 e}+k_{2 e}+i \omega\left(c_{1}+c_{2}\right)\right]-\bar{y}_{2}(\omega)\left(k_{2 e}-i \omega c_{2}\right)=\bar{F}(\omega) \\
-\bar{y}_{1}(\omega)\left(k_{2 e}+i \omega c_{2}\right)+\bar{y}_{2}(\omega)\left[-m_{2} \omega^{2}+k_{2 e}+k_{3 e}+i \omega\left(c_{2}+c_{3}\right)\right]-\bar{y}_{3}(\omega)\left(k_{3 e}+i \omega c_{3}\right)=0 \\
-\bar{y}_{n-1}(\omega)\left(k_{(n-1) e}+i \omega c_{n-1}\right)+\bar{y}_{n-1}(\omega)\left[-m_{n-1} \omega^{2}+k_{(n-1) e}+k_{n e}+i \omega\left(c_{n-1}+c_{n}\right)\right]-y_{n}(\omega)\left(k_{n e}+i \omega c_{n}\right)=0 \\
-y_{n-1}(\omega)\left(k_{n e}-i \omega^{2} c_{n}\right)-y_{n}(\omega)\left(k_{n e}+m_{n} \omega^{2}+i \omega^{2} c_{n}\right)=0
\end{array}\right.
$$

From this system we get the response $\bar{y}_{1}(\omega), \bar{y}_{2}(\omega), \ldots, \bar{y}_{n}(\omega)$.

The frequency response function [3] of the system is given [4] by equation:

$$
H_{1}(\omega)=\frac{\bar{y}_{1}(\omega)}{\bar{F}(\omega)}, H_{2}(\omega)=\frac{\bar{y}_{2}(\omega)}{\bar{F}(\omega)}, \ldots, H_{n}(\omega)=\frac{\bar{y}_{n}(\omega)}{\bar{F}(\omega)} .
$$

The displacement variance [5] of the system under Gaussian white noise excitation can be expressed as:

$$
\left\{\begin{array}{l}
\sigma_{y_{1}}^{2}=R_{y_{1}}(0)=\int_{-\infty}^{\infty}\left|H_{1}(\omega)\right|^{2} m_{1}^{2} S_{0}^{\prime} d \omega=\frac{1}{m_{1}^{2}} \int_{-\infty}^{\infty}\left|H_{1}(\omega)\right|^{2} m_{1}^{2} S_{0}^{\prime} d \omega \\
\sigma_{y_{2}}^{2}=R_{y_{2}}(0)=\int_{-\infty}^{\infty}\left|H_{2}(\omega)\right|^{2} m_{2}^{2} S_{0}^{\prime} d \omega=\frac{1}{m_{2}^{2}} \int_{-\infty}^{\infty}\left|\frac{\square}{H_{2}}(\omega)\right|^{2} m_{2}^{2} S_{0}^{\prime} d \omega \\
\sigma_{y_{n}}^{2}=R_{y_{n}}(0)=\int_{-\infty}^{\infty}\left|H_{n}(\omega)\right|^{2} m_{n}^{2} S_{0}^{\prime} d \omega=\frac{1}{m_{n}^{2}} \int_{-\infty}^{\infty}\left|H_{n}(\omega)\right|^{2} m_{n}^{2} S_{0}^{\prime} d \omega
\end{array}\right.
$$

The power spectral density of response [6] ( in $\left.m^{2} \cdot s\right)$ is given by equation:

$$
S_{1}(\omega)=\left|H_{1}(\omega)\right|^{2} S_{F}=\left|H_{1}(\omega)\right|^{2} m_{1}^{2} S_{0}^{\prime}(\omega)=\left|\frac{H_{1}}{m_{1}}\right|^{2} m_{1}^{2} S_{0}^{\prime}(\omega)=\left|H_{1}(\omega)\right|^{2} S_{0}^{\prime}(\omega), \ldots, S_{n}(\omega)=\left|H_{n}(\omega)\right|^{2} S_{0}^{\prime}(\omega) .(
$$

\section{Numerical results}

In this example, we considered $m_{2}=1.25 \mathrm{~kg}, m_{2}=1.1 \mathrm{~kg}, \quad c_{1}=3.5 \frac{\mathrm{N} \cdot \mathrm{s}}{\mathrm{m}}, \quad c_{2}=3.8 \frac{\mathrm{N} \cdot \mathrm{s}}{\mathrm{m}}$, $k_{1}=28 \frac{N}{m}, k_{2}=25,5 \frac{N}{m}$, the nonlinear component $G(y)=y^{3}(t)$, with the nonlinear factor to control the type and degree of nonlinearity $\alpha=3 m^{-2}$, and wich means that the power spectral density of excitation $S_{0}^{\prime}=0,34 \frac{m^{2}}{s^{3}}$.

$k_{1 e}=k_{1}+3 \alpha \sigma_{y_{1}}^{2}\left(k_{1}+k_{2}\right)-3 \alpha k_{2}\left(\sigma_{y_{1}}^{4}+\sigma_{y_{2}}^{4}\right)\left(\sigma_{y_{1}}^{2}+\sigma_{y_{2}}^{2}\right)^{-1}, k_{2 e}=k_{2}+3 \alpha k_{2}\left(\sigma_{y_{1}}^{4}+\sigma_{y_{2}}^{4}\right)\left(\sigma_{y_{1}}^{2}+\sigma_{y_{2}}^{2}\right)^{-1}$.

Obtain for the response [7]:

$\bar{\eta}_{1}(\omega)=\frac{m_{1} m_{2} \omega^{2}-\left(m_{1}+m_{2}\right) k_{2 e}-i \omega\left(m_{1}+m_{2}\right) c_{2}}{D} \bar{F}(\omega)$ 
$\bar{\eta}_{2}(\omega)=\frac{m_{1} m_{2} \omega^{2}-\left(m_{1} k_{2 e}+m_{2} k_{1 e}+m_{2} k_{2 e}\right) k_{2 e}-i \omega\left(m_{1} c_{2}+m_{2} c_{1}+m_{2} c_{2}\right)}{D} \bar{F}(\omega)$,

where:

$D=m_{1} m_{2} \omega^{4}-\omega^{2}\left(m_{1} k_{2 e}+m_{2} k_{1 e}+m_{2} k_{2 e}+2 c_{2}^{2}+c_{2} c_{1}\right)+k_{1 e} k_{2 e}+i \omega\left(k_{2 e} c_{1}+k_{2 e} c_{2}+k_{1 e} c_{2}+3 c_{2} k_{2 e}\right)-i \omega^{3}\left(m_{1} c_{2}+m_{2} c_{1}+m_{2} c_{2}\right)$.

The frequency response function [8] of the system is give by equation:

$H_{1}(\omega)=\frac{m_{1} m_{2} \omega^{2}-\left(m_{1}+m_{2}\right) k_{2 e}-i \omega\left(m_{1}+m_{2}\right) c_{2}}{D}, \stackrel{\square}{H_{2}}(\omega)=\frac{m_{1} m_{2} \omega^{2}-\left(m_{1} k_{2 e}+m_{2} k_{1 e}+m_{2} k_{2 e}\right)-i \omega\left(m_{1} c_{2}+m_{2} c_{1}+m_{2} c_{2}\right)}{D}$.

The mean square value for the displacement of the system [9] is given by equation:

$\sigma_{y_{1}}^{2}=\pi S_{0}^{\prime} \psi_{1} \psi_{2}^{-1}, \sigma_{y_{2}}^{2}=\pi S_{0}^{\prime} \psi_{3} \psi_{2}^{-1},(25)$

where:

$\psi_{1}=k_{2 e}\left(m_{1}+m_{2}\right)^{2}\left(k_{1 e}\right)^{-1}\left[\left(m_{1} k_{2 e}+m_{2} k_{1 e}+m_{2} k_{2 e}+2 c_{2}^{2}+c_{2} c_{1}\right)\left(m_{1} c_{2}+m_{2} c_{1}+m_{2} c_{2}\right)-\left(k_{2 e} c_{1}+4 k_{2 e} c_{2}+k_{1 e} c_{2}\right) m_{1} m_{2}\right]$

$+\left(m_{1} c_{2}+m_{2} c_{1}+m_{2} c_{2}\right)\left[c_{2}^{2}\left(m_{1}+m_{2}\right)^{2}+2 k_{2 e}\left(m_{1}+m_{2}\right) m_{1} m_{2}\right]+\left(k_{2 e} c_{1}+4 k_{2 e} c_{2}+k_{1 e} c_{2}\right)\left(m_{1} m_{2}\right)^{2}$ (26)

$\psi_{2}=\left(k_{2 e} c_{1}+4 k_{2 e} c_{2}+k_{1 e} c_{2}\right)\left[\left(m_{1} k_{2 e}+m_{2} k_{1 e}+m_{2} k_{2 e}+2 c_{2}^{2}+c_{2} c_{1}\right)\left(m_{1} c_{2}+m_{2} c_{1}+m_{2} c_{2}\right)-\left(k_{2 e} c_{1}+4 k_{2 e} c_{2}\right.\right.$

$\left.\left.+k_{1 e} c_{2}\right) m_{1} m_{2}\right]-\left(k_{1 e} k_{2 e}\right)\left(m_{1} c_{2}+m_{2} c_{1}+m_{2} c_{2}\right)^{2}$,

$\psi_{3}=k_{2 e}\left(m_{1}+m_{2}\right)^{2}\left(k_{1 e}\right)^{-1}\left[\left(m_{1} k_{2 e}+m_{2} k_{1 e}+m_{2} k_{2 e}+2 c_{2}^{2}+c_{2} c_{1}\right)\left(m_{1} c_{2}+m_{2} c_{1}+m_{2} c_{2}\right)-\left(k_{2 e} c_{1}+4 k_{2 e} c_{2}+k_{1 e} c_{2}\right) m_{1} m_{2}\right]+\left(m_{1} c_{2}\right.$ $\left.+m_{2} c_{1}+m_{2} c_{2}\right)\left[\left(m_{1} c_{2}+m_{2} c_{1}+m_{2} c_{2}\right)^{2}-2\left(m_{1} k_{2 e}+m_{2} k_{1 e}+m_{2} k_{2 e}\right)\left(m_{1} m_{2}\right)\right]+\left(k_{2 e} c_{1}+4 k_{2 e} c_{2}+k_{1 e} c_{2}\right)\left(m_{1} m_{2}\right)^{2}$. (28)

Because neglecting wery small terms we get, obtain ${\sigma_{y_{1}}}^{2}=0,144 \mathrm{~m}^{2},{\sigma_{y_{2}}}^{2}=0,196 \mathrm{~m}^{2}$.

The power spectral density of response [10] will be:

$S_{1}(\omega)=\frac{\left[m_{1} m_{2} \omega^{2}-\left(m_{1}+m_{2}\right) k_{2 e}\right]^{2}+\omega^{2}\left[\left(m_{1}+m_{2}\right) c_{2}\right]^{2}}{\lambda_{1}^{2}+\lambda_{2}^{2}} S_{0}^{\prime}(\omega)\left[m^{2} \cdot s\right]$,

$S_{2}(\omega)=\frac{\left[m_{1} m_{2} \omega^{2}-\left(m_{1} k_{2 e}+m_{2} k_{1 e}+m_{2} k_{2 e}\right)\right]^{2}+\omega^{2}\left[\left(m_{1} c_{2}+m_{2} c_{1}+m_{2} c_{2}\right)\right]^{2}}{\lambda_{1}^{2}+\lambda_{2}^{2}} S_{0}^{\prime}(\omega)\left[m^{2} \cdot s\right]$.

where :

$\lambda_{1}=m_{1} m_{2} \omega^{4}-\omega^{2}\left(m_{1} k_{2 e}+m_{2} k_{1 e}+m_{2} k_{2 e}+2 c_{2}^{2}+c_{2} c_{1}\right)+k_{1 e} k_{2 e}, \lambda_{2}=\omega\left(k_{2 e} c_{1}+k_{2 e} c_{2}+k_{1 e} c_{2}+3 c_{2} k_{2 e}\right)-\omega^{3}\left(m_{1} c_{2}+m_{2} c_{1}+m_{2} c_{2}\right),($

We will get the numeric value:

$$
\begin{aligned}
& S_{1}(\omega)=0.34 \frac{\left(1.375 \omega^{2}-61.946\right)^{2}+26.316 \omega^{2}}{\left(1.375 \omega^{4}-108.405 \omega^{2}+797.39\right)^{2}+\left(607.878 \omega-12.78 \omega^{3}\right)^{2}}\left[m^{2} \cdot s\right], \\
& S_{2}(\omega)=0,34 \frac{\left(1.375 \omega^{2}-95.105\right)^{2}+12.78 \omega^{2}}{\left(1.375 \omega^{4}-108.405 \omega^{2}+797.39\right)^{2}+\left(607.878 \omega-12.78 \omega^{3}\right)^{2}}\left[m^{2} \cdot s\right] .
\end{aligned}
$$

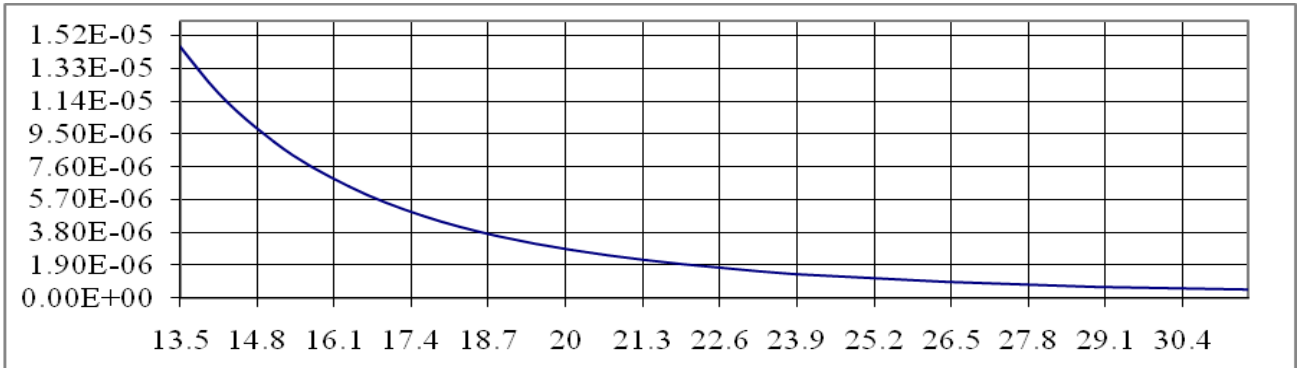

$\omega / 2 \pi$

Figure 2. The power spectral density of the response $S_{1}(\omega)\left[\mathrm{m}^{2} \cdot \mathrm{s}\right]$ for the first oscillator. 


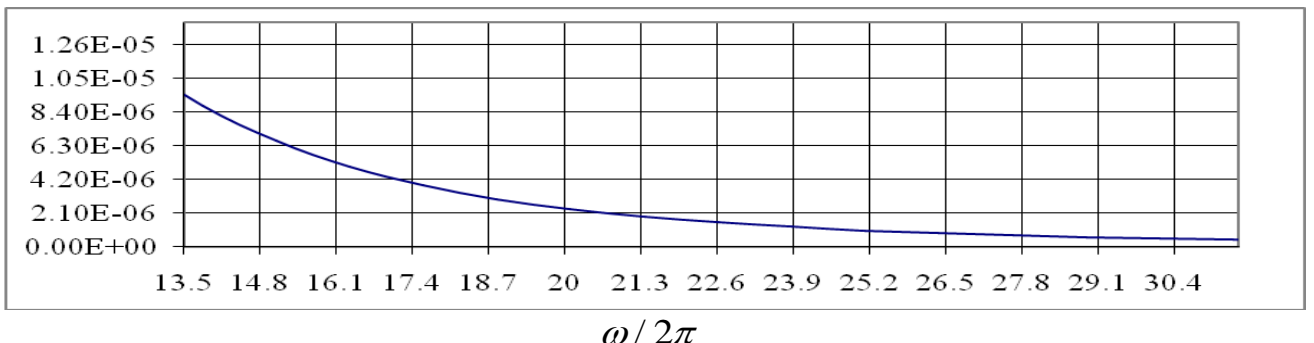

Figure 3. The power spectral density of the response $S_{2}(\omega)\left[\mathrm{m}^{2} \cdot \mathrm{s}\right]$ for the second oscillator.

\section{Remarks}

In the figures 2 and 3 , the power spectral density of the excitation, $S_{1}(\omega)\left[m^{2} \cdot s\right], S_{2}(\omega)\left[m^{2} \cdot s\right]$, is plotted for the differents parameters. Approximate methods for studying non-linear vibrations of beams are important for investigating and designing purposes. In the absence of an exact analytical solution to a nonlinear vibration problem, we wish to find at least an approximate solution. Although both analytical and numerical methods are available for approximate solution of nonlinear vibration problems, the analytical methods are more desirable. Due to the fact that response statistics of such a model can be evaluated analytically, in general, statistical linearization is computationally very efficient.

\section{References}

[1] Roberts J B 1990 Random Vibration and Statistical Linearization (Wiley, Chichester) p 105

[2] Pandrea N.and Parlac S 2000 Mechanical vibrations (Pitesti: Pitesti University Press) p 275

[3] Stan P 2013 Fiability and Durability vol 1 (Târgu Jiu: Târgu Jiu University Press) p 124

[4] Stan P 2013 Termodinamics (Craiova:Craiova University Press) p 97

[5]Crandall S H, Mark W D 1963 Random vibration in mechanical systems (New York:

Academic Press) p 89-98

[6]Fung Y 1955 The Analysis of Dynamic Stresses in Aircraft Structures During Landing as

Nonstationary Random Processes (Wiley, New York) p 449-457

[7] Yang C Y 1986 Random vibration of structures(Wiley, New York) p 122-136

[8] Robson J D 1980 The response relationships of random vibration analysis (Wiley, New York) p312-315

[9] Miles J W 1954 On Structural Fatigue Under Random Loading, .(Wiley, New York) p. 753

[10] Iyengar R N and Dash P K 1976 Random vibration analysis of stochastic time-varying systems (J Sound Vib 45) p 69-89 